\title{
Luttinger liquid with complex forward scattering: Robustness and Berry phase
}

\author{
Balázs Dóra ${ }^{1, *}$ and Roderich Moessner ${ }^{2}$ \\ ${ }^{1}$ Department of Theoretical Physics and BME-MTA Exotic Quantum Phases Research Group, \\ Budapest University of Technology and Economics, 1521 Budapest, Hungary \\ ${ }^{2}$ Max-Planck-Institut für Physik komplexer Systeme, 01187 Dresden, Germany
}

(Received 15 October 2015; revised manuscript received 6 January 2016; published 12 February 2016)

\begin{abstract}
Luttinger liquids (LLs) are one-dimensional systems with well-understood instabilities due to Umklapp or backscattering. We study a generalization of the Luttinger model, which incorporates a time reversal symmetry breaking interaction producing a complex forward scattering amplitude ( $g_{2}$ process). The resulting low energy state is still a LL and belongs to the family of interacting Schulz-Shastry models. Remarkably, it becomes increasingly robust against additional perturbations-for purely imaginary $g_{2}$, both Umklapp and local backscattering are always irrelevant. Changing the phase of the interaction generates a nontrivial Berry phase, with a universal geometric phase difference between ground and a one boson excited state depending only on the LL parameter.
\end{abstract}

DOI: 10.1103/PhysRevB.93.075127

\section{INTRODUCTION}

Landau Fermi liquid theory enjoys great success in describing three-dimensional metals. In particular, through the quasiparticle concept, it explains why interacting fermions behave similarly to a Fermi gas.

However, upon reducing spatial dimensionality, instabilities of the gas become more pronounced and Fermi liquid theory loses its applicability. This is most striking in one dimension, where the conventional quasiparticles break down. They are often replaced by collective bosonic excitations described by Luttinger liquid (LL) theory. LL physics is not restricted to condensed matter physics [1,2] but plays a role whenever confinement to one spatial dimension is strong, e.g., in field theories of high energy physics (e.g., massless Thirring model [3]), cold atoms [4], or the study of black holes [5].

These collective bosonic excitations can further become unstable, in the presence of Umklapp or backscattering due to interactions or impurities, as the LL undergoes a phase transition to a different state: Stabilizing the LL low energy modes represent a difficult task [6].

To harness correlations and entanglement of LL—e.g., in a putative quantum computer [7]-it is desirable for them to be robust to additional perturbations. Besides isolating a LL carefully from its environment, additional stability can either be achieved by prohibiting backscattering through carefully canceling dangerous terms in the LL Hamiltonian; or by adding extra terms, which act to suppress the effects of backscattering while preserving the low energy properties of the LL. Here we follow this latter route by designing a Luttinger liquid with extremely robust collective low energy modes. This is achieved by introducing an interaction breaking time reversal symmetry, which changes from attractive to repulsive depending on whether two particles move towards or away from each other.

We first introduce the Hamiltonian of this generalized LL. We then analyze its properties, demonstrating its immunity with respect to Umklapp and backscattering as a function of

*dora@eik.bme.hu the phase of its complex coupling constant $g_{2}$. We investigate the universality of its geometric phase and finally discuss ingredients for its possible realization.

\section{THE MODEL}

The low energy physics of many types (fermions, bosons, spins) of one-dimensional interacting particles is described by the Luttinger model. We study its generalization to include a complex $g_{2}$ process:

$$
H=\sum_{q \neq 0} \omega(q) b_{q}^{\dagger} b_{q}+\frac{g_{q}}{2} b_{q} b_{-q}+\frac{g_{q}^{*}}{2} b_{q}^{+} b_{-q}^{+},
$$

with $\omega(q)=v|q|, v$ the "sound velocity," $b_{q}^{\dagger}$ the creation operator of a bosonic density wave and $g_{q}=g_{2}|q| \exp (i \varphi)$ parametrizing the interparticle interaction. We have neglected velocity renormalization $[1,8]$. The conventional case, discussed thoroughly in Refs. [1,2,8] corresponds to $\varphi=0$, where forward scattering (small momentum transfer compared to the Fermi momentum $k_{F}$ ) interactions are included. The whole parameter space is covered by arbitrary real values of $g_{2}$ (describing both attractive and repulsive interactions) and restricting the phase to $|\varphi| \leqslant \pi / 2$. Other values of the phase are accounted for by changing the sign of $g_{2}$.

Equation (1) resembles the Bogoliubov Hamiltonian of a Bose-Einstein condensate, with $\varphi$ playing the role of the condensate phase. This phase drops out from most physical observables and correlation functions, being visible primarily in interference probes. We show that for LLs, the innocent-looking phase variable has a profound effect on both correlators and the stability of the LL.

The origin of phase $\varphi$ is readily illustrated for interacting spinless fermions. The conventional real part of the $g_{2}$ process is due to a short-range forward scattering density-density interaction [1], $H_{r e}=g^{\prime} \int d x \rho_{R}(x) \rho_{L}(x)$, where $\rho_{R}(x)=$ : $R^{+}(x) R(x)$ : and $\rho_{R}(x)=: L^{+}(x) L(x)$ : are normal ordered densities, and $R(x) / L(x)$ annihilates a right/left moving particle at point $x$, respectively.

The novel aspect of our work, the imaginary part of the interaction, can be generated from a long range interaction 
between the above densities

$$
H_{i m}=-2 g^{\prime \prime} \int d x \int d y \frac{\rho_{R}(x) \rho_{L}(y)}{x-y} .
$$

This breaks time reversal invariance and has the same scaling dimension (also marginal) as the above $g^{\prime}$ process. Therefore they should be considered on equal footing.

This interaction is repulsive/attractive when two particles move away from/towards each other (or the opposite for both, depending on the sign of $g^{\prime \prime}$ ). In other words, the repulsive or attractive nature of the interaction depends on the relative motion of the interacting pair. The self-energy correction of $H_{i m}$ from diagrammatics gives the same LL behavior as the $H_{r e}$. However, singular vertex corrections are absent, in sharp contrast to the conventional case, see below.

Bosonizing $H_{i m}$ following Ref. [1] yields imaginary $g_{2}$ :

$$
H_{i m}=i g^{\prime \prime} \sum_{q \neq 0} \frac{|q|}{2}\left(b_{q}^{+} b_{-q}^{+}-b_{q} b_{-q}\right) \text {. }
$$

Keeping both $H_{r e}$ and $H_{i m}$ gives Eq. (1) with $g_{2} \cos \varphi=g^{\prime}$ and $g_{2} \sin \varphi=g^{\prime \prime}$.

The phase can be "gauged away" from Eq. (1) by a unitary transformation leaving the eigenenergies and the renormalized velocity $\sqrt{v^{2}-g_{2}^{2}}$ unchanged, with LL parameter $K=\sqrt{\left(v-g_{2}\right) /\left(v+g_{2}\right)}$. The sign change of $g_{2}$ amounts to $K \rightarrow 1 / K$ change. Equation (1) is diagonalized by a Bogoliubov rotation to bosonic operators $c_{q}$ :

$$
b_{q}=\frac{K+1}{2 \sqrt{K}} c_{q}+\exp (i \varphi) \frac{K-1}{2 \sqrt{K}} c_{-q}^{+},
$$

yielding $H=\sum_{q \neq 0} \sqrt{v^{2}-g_{2}^{2}}|q| c_{q}^{+} c_{q}$. The essential change with respect to the conventional case is the phase factor in Eq. (4).

\section{CORRELATION FUNCTIONS}

While the spectrum does not change, correlation functions do. Let us consider an underlying spinless fermion field which decomposes into right- and left-going parts, $\Psi(x)=$ $e^{i k_{F} x} R(x)+e^{-i k_{F} x} L(x)$. To evaluate the right-going Green function

$$
G_{R}(x, t) \equiv\left\langle R^{+}(x, t) R(0, t)\right\rangle,
$$

$R(x)$ is expressed in terms of the real space version of the $b$ operators via [1] $R(x)=\frac{1}{\sqrt{2 \pi \alpha}} \exp [i(\phi(x)-\theta(x))]$ with $[\phi(x), \theta(y)]=i \frac{\pi}{2} \operatorname{sign}(y-x)$ the commutation relation of the dual fields. These are related to the $b$ bosons as $\phi(x)=\sum_{q} \sqrt{2 \pi /|q| L} e^{i q x-\alpha|q| / 2} b_{q}+$ H.c. and $\theta(x)=$ $\sum_{q} \operatorname{sign} q \sqrt{2 \pi /|q| L} e^{i q x-\alpha|q| / 2} b_{q}+$ H.c. and $\alpha$ is the short distance cutoff. Standard analysis [1,2] yields $G_{R}(x, t) \sim$ $|x|^{-\eta_{R}}$ with

$$
\eta_{R}=\frac{K+K^{-1}}{2}
$$

independent of $\varphi$. The left-movers behave identically. Nonetheless, for the correlators of the dual fields, we find

$$
\begin{gathered}
\left\langle(\phi(x, t)-\phi(0, t))^{2}\right\rangle=\eta_{\phi} \ln (x), \\
\eta_{\phi}=K \cos ^{2}(\varphi / 2)+(1 / K) \sin ^{2}(\varphi / 2) .
\end{gathered}
$$

Conventional duality of LLs for $\left\langle(\theta(x, t)-\theta(0, t))^{2}\right\rangle=$ $\eta_{\theta} \ln (x)$, using the $K \rightarrow 1 / K$ change for $\eta_{\theta}$, gives

$$
\eta_{\theta}=(1 / K) \cos ^{2}(\varphi / 2)+K \sin ^{2}(\varphi / 2) .
$$

The dominant instabilities for spinless fermions are expected in the $2 k_{F}$ charge density wave or in the superconducting channel, with order parameters $O_{C D W}=R^{+}(x) L(x) \sim$ $\exp (-i 2 \phi(x))$ and $O_{S C}=R(x) L(x) \sim \exp (-2 i \theta(x))$, respectively. Their correlation functions decay with respective exponents $-2 \eta_{\phi}$ and $-2 \eta_{\theta}$. For $\cos (\varphi)>0$, the conventional conclusions stand [1]: for $0<K<1$ (attractive interactions), the SC instability dominates, while for repulsive $K>1$, density wave ordering is favored. For $\cos (\varphi)<0$, however, the reverse is the case.

In between, for $\varphi= \pm \pi / 2$, the interaction term is purely imaginary, and the exponents are equal, $2 \eta_{\phi}=2 \eta_{\theta}=K+$ $K^{-1}>2$, exactly twice $\eta_{R}$ of the single particle Green function, Eq. (6). This indicates the absence of singular vertex corrections due to the peculiar attractive-repulsive behavior of the interaction, Eq. (2). The correlation functions thus decay with an exponent bigger than 2 , implying a faster decay to zero than in the noninteracting case in both particle-hole and Cooper channels. As a result, these instabilities are excluded.

\section{UMKLAPP AND BACKSCATTERING}

To assess the stability of this LL, one has to consider further scattering processes which could induce a gap. One is Umklapp scattering, arising at half filling for spinless particles:

$$
H_{u}=\frac{g_{u}}{(2 \pi \alpha)^{2}} \int d x \cos (4 \phi(x)) .
$$

From Eq. (7), $\langle\cos (4 \phi(x)) \cos (4 \phi(y))\rangle \sim|x-y|^{8 \eta_{\phi}}$, yielding scaling dimension $4 \eta_{\phi}$ [2]. In $1+1=2$ space-time dimensions of the model, the Umklapp term thus is relevant for $4 \eta_{\phi}<$ 2. This reproduces the result $K<1 / 2$ for a conventional LL $(\varphi=0)[1]$. For the general case, the perturbation can only be relevant $\left(\eta_{\phi}<1 / 2\right)$ for $|\varphi|<\pi / 6$, otherwise it is irrelevant regardless of the value of $K$. For spinful fermions, e.g. in the charge sector of the Hubbard model, Umklapp scattering gives rise to a term with $\cos (\sqrt{8} \phi(x))$, relevant for $\eta_{\phi}<1$.

The $K-\varphi$ phase diagram for these two cases is plotted in Fig. 1. For purely imaginary interaction, $\varphi= \pm \pi / 2$, these sine-Gordon-like terms are all irrelevant and the LL is stable for any $K$, even at commensurate filling. This is due to the lack of vertex corrections from Eq. (2).

Local perturbations such as potential scattering can modify the transport properties of a LL. Depending on the LL parameter, it can flow to strong coupling and effectively cut the LL in two, or to zero and disappear from the low energy dynamics. For the spinless case, local backscattering takes the 


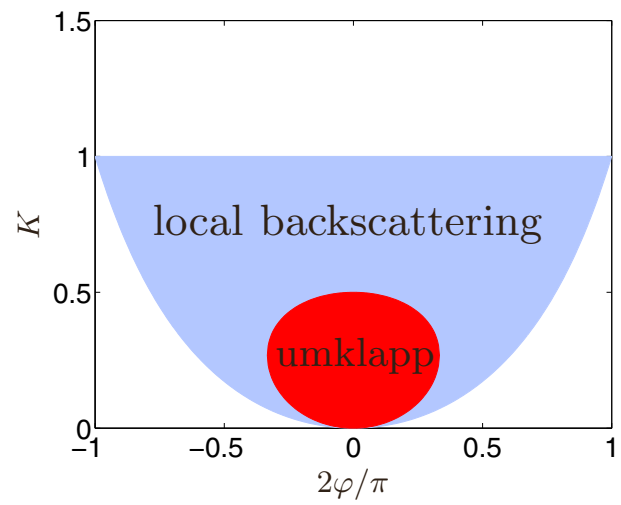

FIG. 1. The $K-\varphi$ phase diagram for spinless fermions, the colored regions denote the relevance of the Umklapp processes (red) and local backscattering (blue). The latter region also denotes the relevance of the Umklapp scattering for spinful fermions in, e.g., the Hubbard model. For cosine terms containing $\theta, K$ should be changed to $1 / K$.

form

$$
H_{b s}=\frac{g_{b s}}{\pi \alpha} \cos (2 \phi(0)) .
$$

Since this interaction is spatially localized, only its temporal fluctuation needs to be considered [2]. It is relevant for $\eta_{\phi}<1$ (as for the conventional LL [1,2]), just as Umklapp scattering in the spinful case, Fig. 1.

\section{GEOMETRIC PHASE RELATED TO $\varphi$}

We now turn to the properties treating the phase $\varphi$ as a tunable degree of freedom. A noninteracting fermionic version of Eq. (1) was considered in Refs. [9,10], and its Berry phase signaled quantum phase transitions. Our model, on the other hand, contains interacting fermionic degrees of freedom and is always at criticality with continuously varying critical exponents so that its geometric phase can be sensitive to both criticality and interactions.

In the Luttinger model, a given momentum $q$ mode only interacts with its $-q$ partner. It therefore is suffices to analyze a single $(q,-q)$ pair in Eq. (1). This is identical to a quantum parametric amplifier [11] with a time dependent $\varphi$. The $b_{q}^{+} b_{q}-$ $b_{-q}^{+} b_{-q}$ being an unbounded constant of motion, an appropriate $\varphi(t)$ can enhance the boson occupation numbers and yield squeezing [12]. Here we investigate the effect of an adiabatic change of this phase in Eq. (1) from 0 to $2 \pi$. The calculation of the geometric phase related to this cycle follows similar steps in the Dicke model [13]. The phase is induced in Eq. (1) by the unitary transformation

$$
U_{R}=\exp \left(-i \frac{\varphi}{2} \sum_{q \neq 0} b_{q}^{\dagger} b_{q}\right),
$$

with concomitant ground state wave function transformation $|\Psi(\varphi)\rangle=U_{R}|\Psi(0)\rangle$. Thence,

$$
\gamma_{g}=i \int_{0}^{2 \pi} d \varphi\left\langle\Psi(\varphi)\left|\partial_{\varphi}\right| \Psi(\varphi)\right\rangle=\pi \sum_{q \neq 0}\left\langle b_{q}^{\dagger} b_{q}\right\rangle .
$$

For a given mode, the geometric phase is independent of momentum from Eq. (4):

$$
\tilde{\gamma}=\frac{\pi}{4}\left(K+\frac{1}{K}-2\right) .
$$

The overall geometric phase $\gamma_{g}=L \tilde{\gamma} / \pi \alpha$ is not universal as it depends on the high energy cutoff $\alpha$.

The elementary excitations in a LL lose their original fermionic character and statistically transmute into bosons [2]. It is thus interesting to consider the relative geometric phase between a nonzero momentum excited state with one boson and the ground states [9], given by the difference of the respective Berry phases. A one-boson excited state with momentum $k$ is $c_{k}^{+}|\Psi(\varphi)\rangle$. Then

$$
\gamma_{e g}=\gamma_{e}-\gamma_{g}=i \int_{0}^{2 \pi} d \varphi\left\langle\Psi(\varphi)\left|c_{k} \partial_{\varphi} c_{k}^{+}\right| \Psi(\varphi)\right\rangle=-\tilde{\gamma}
$$

is universal and depends only on the LL parameter $K$. This is not topological but geometric in nature and can be tuned arbitrarily by changing the strength of the interaction. The Berry phase difference between any two one-boson excited states vanishes.

$\gamma_{g}$ also follows from the fact that the terms in the Hamiltonian for each $(q,-q)$ pair are the generators [14] of the $\mathrm{SU}(1,1)$ Lie algebra. In particular, $J_{z}(q)=\left(b_{q}^{+} b_{q}+\right.$ $\left.b_{-q} b_{-q}^{+}\right) / 2, J_{+}(q)=b_{q}^{+} b_{-q}^{+}, J_{-}(q)=b_{q} b_{-q}$ are the generators of a SU(1,1) Lie algebra with commutation relation $\left[J_{+}(q), J_{-}(q)\right]=-2 J_{z}(q),\left[J_{z}(q), J_{ \pm}(q)\right]= \pm J_{ \pm}(q)$. The geometric phase for general time dependent parameters for the $\mathrm{SU}(1,1)$ case were calculated in Refs. [11,15], which also gives Eq. (14). Due to the bosonic algebra in LLs, the geometric phase is related to the surface area on the unit hyperboloid (and not the unit sphere, as in the case for fermions) enclosed by the adiabatic path traced out by $U_{R}(t)$.

\section{CONNECTION TO INTERACTING GAUGE THEORY}

Gauge fields and their role in statistical transmutation in various dimensions are a fascinating subject of research [16]. A gauge potential enters into the Hamiltonian of a LL, written in terms of the dual fields as

$$
H=\frac{\tilde{v}}{2 \pi} \int d x \frac{1}{\tilde{K}}\left[\partial_{x} \phi(x)\right]^{2}+\tilde{K}[\Pi(x)-A(x)]^{2},
$$

where $\Pi(x)=\partial_{x} \theta(x) / \pi, \tilde{K}$ and $\tilde{v}$ are LL parameter and renormalized velocity, respectively. For a particular long range density dependent gauge potential of strength $v$,

$$
A(y)=v \int d x \frac{\partial_{x} \phi(x)}{x-y},
$$

the second term in Eq. (15) generates $H^{\prime}$ in Eq. (2), and $\partial_{x} \phi(x)=-\pi\left[\rho_{R}(x)+\rho_{L}(x)\right]$ is the density operator for long wavelength excitations.

Equation (15) belongs to the family of Schulz-Shastry models [17,18]. $A(y)$ appears in Ref. [19] and is also common in the factorization of the Calogero-Sutherland model [20]. It represents the Hilbert transform of the charge density, acting over the wave function as the current operator [21]. $A(y)$ can always be unitarily transformed away [17] in 1D from Eq. (15) 
to yield a conventional LL form,

$$
\exp (i S) \Pi(y) \exp (-i S)=\Pi(y)+v \int d x \frac{\partial_{x} \phi(x)}{x-y}
$$

with $\quad S=v \int d x_{1} \int d x_{2} \phi\left(x_{1}\right) \phi\left(x_{2}\right) /\left(x_{1}-x_{2}\right)^{2}=$ $v \int d x_{1} \int d x_{2} \partial_{x} \phi\left(x_{1}\right) \partial_{x} \phi\left(x_{2}\right) \ln \left|x_{1}-x_{2}\right|$. Note that the latter form of $S$ describes a logarithmic long range interaction between the densities.

Equation (17) can be regarded as a generalized Jordan-Wigner transformation [17,18,22], $\theta(y) \rightarrow \theta(y)-$ $\pi v \int d x \ln |x-y| \partial_{x} \phi(x)$. Unlike a local vector potential $A_{l}(x)=v \partial_{x} \phi(x)$, which yields anyons in $1 \mathrm{D}$, the present transformation does not change the statistics of the original fields.

\section{RELATION TO EXPERIMENTS}

Here we list some ingredients for obtaining a LL with complex $g_{2}$ and give pointers for how they may perhaps be realized. Since the gauge potential in 1D [17] can be transformed away, the thermodynamics of Eqs. (1) and (15) is identical to that of a conventional LL. However, the unitary transformation has to be reinserted to compute correlators. This suggests one simulation strategy for the gauge transformed system in a quench experiment, since the unitary transformation can formally be identified with a time evolution operator: by preparing a one dimensional system in its LL ground state [4], adding a spatially long range logarithmic interaction, for a duration proportional to the strength of the gauge field $\nu$, mimics $S$ and the unitary transformation in Eq. (17). A logarithmic interaction can, e.g., be approximated in dielectric films endowed with permeability much higher than the surrounding medium [23]. The equal time correlation functions evaluated with the quenched wave function would then be identical to those from Eq. (15). In particular, starting from a conventional LL wave function $\left|\Psi_{L L}\right\rangle$ and introducing a logarithmic long range interaction between the densities with strength $J$ through a sudden quench, the time evolution operator reads as

$$
U(t)=\exp \left(-i t J \int d x_{1} \int d x_{2} n\left(x_{1}\right) n\left(x_{2}\right) \ln \left|x_{1}-x_{2}\right|\right),
$$

$|\Psi(t)\rangle=U(t)\left|\Psi_{L L}\right\rangle$. The expectation values of physical quantities $O$ are

$$
\langle\Psi(t)|O| \Psi(t)\rangle .
$$

Thus, through a quench experiment, equal time correlators of Eq. (15) are simulated. The duration of the quench time and interaction strength, $t J$ controls the strength of the gauge field $v$.

Alternatively, a density dependent, possibly long range gauge potential, Eq. (16), can in principle be created in a cold atomic setting using ideas similar to Refs. [24-27]. More directly, a repulsive or attractive interaction for particles moving away from or towards each other is reminiscent of the physics of Doppler cooling: The frequency of a photon emitted by one atom as seen by the other depends on the direction of their velocity difference though the Doppler effect, so that frequency-dependent absorption could generate the above effect.

Finally, coupling to chiral fields of fermionic [28], bosonic, or other origin also provides the desired interaction $H^{\prime}$. On integrating out the chiral field $\gamma$, which couples as $\left[\rho_{R}(x)+i \rho_{L}(x)\right] \gamma(x)+$ H.c., a long range interaction between the densities arises, mediated by the field propagator $\left\langle\gamma\left(x, t_{1}\right) \gamma^{+}\left(y, t_{2}\right)\right\rangle \sim i /\left(x-y+v_{\gamma}\left(t_{1}-t_{2}\right)\right)$. Assuming the propagation velocity $v_{\gamma}$ of the chiral fields is much larger than the Fermi velocity $v$ in a LL, this yields an instantaneous, nonretarded long range interaction of the desired form, analogously to the instantaneous Coulomb repulsion from photons.

While the experimental obstacles appear formidable, it seems clear that there is no fundamental barrier to realizing our generalized LL. Given its interesting properties, in particular its unusual stability, we hope that it will prove to be worth the requisite effort.

\section{ACKNOWLEDGMENTS}

This research was supported by the Hungarian Scientific Research Funds Nos. K101244, K105149, K108676, by the Bolyai Program of the HAS, and by Helmholtz VI 521 "New States of Matter and Their Excitations."
[1] T. Giamarchi, Quantum Physics in One Dimension (Oxford University Press, Oxford, 2004).

[2] A. O. Gogolin, A. A. Nersesyan, and A. M. Tsvelik, Bosonization and Strongly Correlated Systems (Cambridge University Press, Cambridge, 1998).

[3] W. E. Thirring, Ann. Phys. 3, 91 (1958).

[4] M. A. Cazalilla, R. Citro, T. Giamarchi, E. Orignac, and M. Rigol, Rev. Mod. Phys. 83, 1405 (2011).

[5] V. Balasubramanian, I. García-Etxebarria, F. Larsen, and J. Simón, Phys. Rev. D 84, 126012 (2011).

[6] E. Plamadeala, M. Mulligan, and C. Nayak, Phys. Rev. B 90, 241101 (2014).
[7] M. Nielsen and I. Chuang, Quantum Computation and Quantum Information (Cambridge University Press, Cambridge, 2000).

[8] J. Sólyom, Adv. Phys. 28, 201 (1979).

[9] A. C. M. Carollo and J. K. Pachos, Phys. Rev. Lett. 95, 157203 (2005).

[10] A. Hamma, arXiv:quant-ph/0602091.

[11] C. Gerry, in Coherence and Quantum Optics VI, edited by J. Eberly, L. Mandel, and E. Wolf (Plenum Press, New York, 1989), p. 407.

[12] H. J. Carmichael, G. J. Milburn, and D. F. Walls, J. Phys. A 17, 469 (1984).

[13] G. Chen, J. Li, and J.-Q. Liang, Phys. Rev. A 74, 054101 (2006). 
[14] B. Dóra, A. Bácsi, and G. Zaránd, Phys. Rev. B 86, 161109 (2012).

[15] F. Hong-Yi and H. R. Zaidi, Can. J. Phys. 66, 978 (1988).

[16] S. J. Benetton Rabello, Phys. Rev. Lett. 76, 4007 (1996).

[17] H. J. Schulz and B. S. Shastry, Phys. Rev. Lett. 80, 1924 (1998).

[18] K.-V. Pham, M. Gabay, and P. Lederer, EPL 51, 161 (2000).

[19] U. Aglietti, L. Griguolo, R. Jackiw, S.-Y. Pi, and D. Seminara, Phys. Rev. Lett. 77, 4406 (1996).

[20] B. S. Shastry, Phys. Rev. Lett. 69, 164 (1992).

[21] E. H. Fradkin, E. F. Moreno, and F. A. Schaposnik, Nucl. Phys. B 392, 667 (1993).

[22] K. Penc and B. S. Shastry, Phys. Rev. B 65, 155110 (2002).
[23] L. V. Keldysh, Pis'ma Zh. Eksp. Teor. Fiz. 29, 716 (1979) [JETP Lett. 29, 658 (1979)].

[24] T. Keilmann, S. Lanzmich, I. McCulloch, and M. Roncaglia, Nat. Commun. 2, 361 (2011).

[25] M. J. Edmonds, M. Valiente, G. Juzeliūnas, L. Santos, and P. Öhberg, Phys. Rev. Lett. 110, 085301 (2013).

[26] S. Greschner, G. Sun, D. Poletti, and L. Santos, Phys. Rev. Lett. 113, 215303 (2014).

[27] N. Goldman, G. Juzeliūnas, P. Öhberg, and I. B. Spielman, Rep. Prog. Phys. 77, 126401 (2014).

[28] N. Y. Yao, C. R. Laumann, A. V. Gorshkov, H. Weimer, L. Jiang, J. I. Cirac, P. Zoller, and M. D. Lukin, Nat. Commun. 4, 1585 (2013). 